\title{
Wie TIAs das Leben verkürzen
}

\begin{abstract}
An einer Transitorischen ischämischen Attacke (TIA) stirbt man nicht. Die Symptome bilden sich innerhalb von Stunden zurück. Mit Bild gebenden Verfahren ist meist keine Veränderung im Gehirn mehr zu erkennen. Bisher gibt es kaum Daten darüber, ob eine TIA „nur" einen Warnhinweis auf eine erhöhte Schlaganfallgefährdung darstellt oder ob sich die Attacke selbst schon auf die Überlebenszeit auswirkt.
\end{abstract}

- Wissenschaftler der New South Wales University in Australien ermittelten 22157 Erwachsene, die zwischen Juli 2000 und Juni 2007 wegen einer TIA ins Krankenhaus kamen, und verfolgten ihre Krankengeschichte mindestens zwei Jahre lang (im Schnitt gut vier Jahre). Zudem werteten sie das Sterberegister für diese Zeit aus und verglichen die To- deszahlen ihrer Teilnehmergruppe mit denen der Allgemeinbevölkerung. Das Durchschnittsalter lag für die weiblichen Patienten bei 78, das Alter der Männer bei 73 Jahren. Knapp 24\% waren jünger als 65 und knapp 20\% älter als 85 Jahre.

Ein Jahr nach dem Ereignis lebten noch $91,5 \%$ der TIA-Patienten - in der Kontrollgruppe waren es $95 \%$. Nach fünf Jahren lag die Überlebensrate für TIA-Patienten 13,2\% niedriger als in der Vergleichsgruppe. Zum Studienende nach neun Jahren lag die Lebenserwartung der TIA-Patienten sogar 20\% unter der der Vergleichsgruppe.

Im ersten Jahr nach der TIA hatten Männer eine höhere Sterblichkeit als Frauen. Nach dem ersten Jahr wurde dieser Geschlechtsunterschied nicht mehr beobachtet. Bei Patienten, die zum Zeitpunkt der TIA unter 50 Jahre alt wa- ren, hatte das Ereignis nur einen geringen Einfluss auf die Sterblichkeit. Bei Patienten über 65 Jahren wirkte sich die TIA jedoch signifikant lebensverkürzend aus.

\section{Kommentar}

Die Studie ist die erste, die die Langzeiteffekte der TIA auf das Überleben quantifiziert. Sie fand nur minimale Effekte, wenn die TIA-Patienten jünger als 50 Jahre alt waren, aber eine signifikante Reduktion in der Lebenserwartung, wenn die TIA-Patienten älter als 65 Jahre waren. Von einer konsequenten Schlaganfallprävention nach TIA sollte also in erster Linie diese Altersgruppe profitieren.

K. MALBERG

- M. Gattellari et al

Relative Survival After Transient Ischaemic Attack. Results From the A Program of Research Informing Stroke Management (PRISM) Study Published online before print Nov 10, 2011; doi: 10.1111/StrokeAHA.111.6362.33 